\title{
Current Status and Future Perspectives of Axillary Management in the Neoadjuvant Setting
}

\author{
Thorsten Kühn $^{a} \quad$ Jean-Marc Classe ${ }^{b} \quad$ Oreste David Gentilinic \\ Florentia Peintinger $^{e, f}$ Jana de Boniface ${ }^{g, h}$ \\ ${ }^{a}$ Department of Gynecology and Obstetrics, Klinikum Esslingen, Esslingen, Germany; \\ bInstitut de Cancerologie de I'Ouest, Centre Gauducheau, Nantes, France; \\ ${ }^{\mathrm{c} B r e a s t}$ Surgery Unit, San Raffaele University and Research Hospital, Milan, Italy; \\ ${ }^{\mathrm{d}}$ Humanitas Cancer Center, Milan, Italy; \\ e Department of Gynecology and Obstetrics, General Hospital Leoben, Leoben, Austria; \\ ${ }^{f}$ Institute of Pathology, Medical University of Graz, Graz, Austria; \\ gDepartment of Surgery, Capio St. Göran's Hospital, Stockholm, Sweden; \\ h Department of Molecular Medicine and Surgery, Karolinska Institutet, Stockholm, Sweden
}

\section{Keywords}

Breast cancer · Neoadjuvant chemotherapy - Sentinel lymph node biopsy · Axillary surgery

\section{Summary}

Axillary surgery has undergone considerable changes in recent years, especially in relation to patients who undergo neoadjuvant chemotherapy (NACT). Due to constantly decreasing rates of recurrence and death from breast cancer, modern surgical strategies aim at de-escalating the extent of local treatment and avoiding unnecessary procedures. This relates especially to lymph node surgery which is associated with considerable morbidity. In patients who initially present with clinically node-negative disease, sentinel lymph node biopsy (SLNB) is increasingly performed after NACT. The determination of the post-NACT nodal status does not only spare patients from additional surgery but also allows the assessment of pathologic complete response which is increasingly becoming an important tool for treatment planning. Since more than $70 \%$ of these patients have a ypNO status after NACT, future trials will aim to identify patients who might be spared any axillary surgery after NACT. In patients who initially present with positive lymph nodes, the success rates of SLNB in terms of detection and accuracy are less favorable compared to those in patients who undergo primary surgery. The clinical significance of this is unclear. To reduce unnecessary axillary dissection in patients with cN1ycN0 status, prospective outcome data after SLNB without further lymph node re- moval are urgently needed. Improvements in surgical technique by localizing positive nodes at the time of diagnosis and removing them in a targeted surgical procedure (targeted axillary dissection) are under evaluation. Risk assessment and patient selection (including gene expression profiles) might be other ways of safely omitting axillary dissection.

(c) 2018 S. Karger GmbH, Freiburg

\section{Introduction}

Neoadjuvant chemotherapy (NACT) was initially introduced to downstage locally advanced breast cancers and to allow surgery in primarily inoperable cases. Subsequent randomized trials showed that NACT reduces the extent of breast surgery and allows higher rates of breast-conserving surgery (BCS) without jeopardizing long-term survival. Results from recent meta-analyses showed a close relationship between response to NACT and overall survival (OS). Increasing evidence suggests that the effect of NACT on the breast and lymph nodes is a further important tool to tailor postNACT locoregional and systemic treatment decisions.

The role of axillary surgery in the neoadjuvant setting has been debated in recent years and is undergoing continuous modifications relating to the diagnostic versus potentially therapeutic roles of sentinel lymph node biopsy (SLNB) and axillary lymph node dissection (ALND). Axillary interventions in the neoadjuvant setting strongly depend on the clinical lymph node status (cN0 vs. $\mathrm{cN} 1$ ) due to the therapeutic consequences of the assessment outcome and the varying success rates of SLNB.

\section{KARGER \\ Fax +497614520714

(๑) 2018 S. Karger GmbH, Freiburg
Prof. Dr. med. Thorsten Kühn 


\section{Current Standard of SLNB in cNO Patients}

In patients who present with clinically unsuspicious nodes, axillary staging can be performed before or after systemic treatment. One argument in favor of performing SLNB before NACT is that the untreated lymph node status is still considered as one of the strongest prognostic factors. Importantly, adjuvant radiotherapy (RT) (irradiation of extra-axillary lymph nodes and/or chest wall) is based on the primary lymph node status, while systemic treatment decisions are rather based on tumor biology and predictive factors. This applies especially to patients undergoing NACT. Furthermore, clinically occult lymph node involvement of 1-2 sentinel lymph nodes (SLN) pre-NACT will rarely turn the surgical decision towards an ALND, especially in patients who undergo irradiation of the breast.

Three arguments support the performance of SLNB after NACT. Firstly, the patient is spared a separate surgical procedure if axillary staging prior to NACT is omitted. Secondly, the response to NACT, being a strong predictor for OS, is determined by assessing tumor response to both the breast and the lymph nodes. Pathologic complete response ( $\mathrm{pCR}$ ), a widely used parameter to assess the effect of NACT, is defined as the absence of residual tumor in the breast and the lymph nodes (ypT0ypN0). If SLNB is performed before NACT, the assessment of a nodal pCR after NACT is futile. The third argument refers to the fact that $20-40 \%$ of patients will convert from a cN0pN1 stage to ypN0 and can be spared additional regional treatment.

The success rates for SLNB after NACT in initially cN0 patients in terms of detection rate (DR) and accuracy (false-negative rate (FNR)) are very similar to those in pre-NACT SLNB. In the GANEA 1 trial, the post-NACT DR of $94.6 \%$ in $\mathrm{cN} 0$ patients was clearly superior to that of $81.5 \%$ in patients with initially positive lymph nodes (cN1). The corresponding FNRs were 9.4 and 15\%, respectively [1]. Similar results were obtained in a retrospective study from the MD Anderson Cancer Center [2]: Among 3,746 cN0 patients who underwent SLNB and ALND, 3,171 received upfront surgery and 575 received NACT. The reported DRs were $99 \%$ versus $97 \%$ and FNRs $6 \%$ versus $4 \%$. In summary, the success rates of post-NACT SLNB in $\mathrm{CN} 0$ patients are very similar to those in patients undergoing primary surgery. In the $\mathrm{cN} 1$ patient, however, SLNB post-NACT shows inferior success rates as is shown below.

In contrast to the increasing body of evidence showing excellent success rates of SLNB after NACT for cN0 patients, outcome data regarding disease-free survival (DFS) and OS are still sparse. The GANEA 2 trial was the first to provide prospectively collected locoregional relapse rates for cN0 patients undergoing NACT [3]. Among 590 patients, 574 (97\%) had successful SLN identification. Of these, 432 patients had a negative SLNB, 418 of whom had no further axillary surgery. Axillary relapse was observed in only 1 $(0.2 \%)$ patient after a follow-up of 3 years. In a retrospective study of $157 \mathrm{cN} 0 \mathrm{ypN} 0(\mathrm{sn})$ patients without completion ALND, Galimberti et al. [4] observed only $1(0.6 \%)$ axillary relapse after 5 years.

In summary, SLNB after NACT for cN0 patients is feasible, accurate, and safe. There is still some debate regarding the clinical significance of SLNB performed before or after NACT, which may explain different recommendations in international guidelines. SLNB after NACT is the preferred procedure according to the NCCN and the German S3 and AGO guidelines [5-7]. In contrast, in Sweden, SLNB is recommended before NACT [8]. At the St. Gallen conference in 2017, a majority (60\%) of the panelists voted for SLNB after NACT, 20\% preferred SLNB before NACT, and $16.7 \%$ felt that SLNB should be performed in either setting [9].

\section{Axillary Management in cNO in the Neoadjuvant Setting -}

Future Perspectives

It is an important objective in breast cancer treatment to improve the effectiveness of therapy by means of an interdisciplinary approach, reduce treatment-related side effects, and avoid unnecessary procedures. In this context, 2 new issues regarding axillary surgery will need to be assessed in the future.

\section{Can SLNB after NACT Be Omitted in Certain Subgroups of Patients?}

A recent study from MD Anderson showed a close relationship between tumor response after NACT in the breast (pCR-breast) and the axilla (pCR-nodes) [10]. A strong association between response and intrinsic subtype of the primary tumor was also observed. Out of 116 patients with triple-negative (TN) or human epidermal growth factor receptor 2(HER2)-positive tumors with $\mathrm{T} 1-2 / \mathrm{cN} 0$ disease who achieved a complete response in the breast, none had evidence of axillary lymph node metastases. These data were confirmed by 2 presentations at the European Breast Cancer Conference 2018: Data presented by Siso et al. [11] showed a pCRbreast in 23 (42.5\%) of $54 \mathrm{cN} 0$ women with TN and HER2-positive tumors who underwent NACT; none of these patients had nodal disease after systemic treatment. Similar results were obtained by van der Noorda et al. [12]; among 82 patients with a pCR-breast after NACT (all subtypes included), none had a positive SLN. Future studies will therefore focus on patients in whom axillary surgery can be completely omitted after primary systemic treatment.

\section{Can ALND Be Replaced by RT in Patients with cN0ypN1(sn)} Status?

Around $25 \%$ of clinically node-negative patients will have a positive SLN after NACT (cN0ypN1) [3]. These patients are still recommended ALND today. However, ALND does not provide any relevant diagnostic information that might tailor further treatment decisions in these patients, and the therapeutic significance of ALND in this setting is still unknown. In the adjuvant setting, the AMAROS trial showed similar regional control rates and equivalent survival rates for axillary RT and ALND [13]. Morbidity, however, was significantly reduced after RT. Replacement of ALND by $\mathrm{RT}$ could therefore be a viable option in $\mathrm{cN} 0$ ypN1 (sn) patients but requires further investigation. 


\section{Current Standard in cN1 Patients}

In patients who initially present with biopsy-proven positive lymph nodes, the $\mathrm{pN}$ status as a prognostic factor is already determined. The clinical benefit of replacing ALND after NACT with an SLNB in these patients is obvious, since $20-40 \%$ of women convert to a ypN0 status and therefore do not derive any benefit from ALND. Instead, they are exposed to the considerable morbidity associated with the procedure. There are, however, some concerns regarding the reliability of SLNB in terms of feasibility (DR), accuracy (FNR), and oncologic safety (regional recurrences (RR)) in this setting.

At first, performing SLNB after NACT in patients initially presenting with clinical node positivity $(\mathrm{cN} 1)$ was questioned due to unacceptably low detection rates. Suspected to be caused by fibrotic changes in lymphatic channels, detection rates are significantly lower in $\mathrm{cN} 1$ compared to clinically node-negative (cN1) patients [14]. A pooled detection rate of $88.2 \%$ was reported in an early systematic review [13]. As reported in the German SENTINA trial, the dual tracer method combining blue dye and radiocolloid was superior to radiocolloid alone regarding identification rates in initially $\mathrm{cN}+$ patients converting to $\mathrm{ycN}$ - (group C) with 87.8 versus $77.4 \%$. This remained statistically significant even in multivariable testing [15]. In the ACOSOG Z1071 trial, however, the detection rate in $\mathrm{cN}+$ patients was $92.7 \%$ after NACT [16].

An even more important concern is that of the FNR of SLNB after NACT. While the median FNR in primary surgery was reported to be less than $10 \%$ [6], the FNRs after NACT in initially $\mathrm{cN}+$ patients are consistently around $14 \%$ [13, 15-18]. A false-negative SLNB after NACT poses an entirely different theoretic risk than a false-negative SLNB before NACT due to the obvious therapy resistance of a residual lymph node metastasis which is then (as left undetected) only potentially being handled by additional RT. Both ACOSOG Z1071 [16] and the SN FNAC study [19] predefined SLNB as successful in the case of an FNR of less than 10\%, and the outcome was therefore considered negative in both. ACOSOG Z1071, SENTINA, and the Swedish trial showed clearly improved FNRs with an increasing number of identified SLNs; FNRs in the case of at least 3 SLNs removed were as low as 7 and 9\%, respectively, and $4 \%$ in the case of at least 2 SLNs in the Swedish study. In light of the clear definition of an SLN as being blue and/ or radioactive, however, it does not seem acceptable to increase SLN harvest by simply removing another random node at SLNB; thus, only those patients having truly multiple SLNs may be assigned the fortunate subgroup with an acceptable FNR of less than $10 \%$. This group, however, made up only 56 and $34 \%$ of the total $\mathrm{cN}+$ study population in the ACOSOG Z1071 and the SENTINA trial, respectively. In the Swedish study, 11 out of 13 false-negative cases had only 1 SLN identified.

To further improve the feasibility (DR) and reliability (FNR) of SLNB after NACT in cN1 patients, technical improvement of the SLNB procedure was suggested $[15,16]$, and targeted axillary dissection (TAD) was introduced in this context. This technique is based on the concept of marking positive lymph nodes before the start of NACT and then locating and removing those marked nodes in a targeted procedure after systemic treatment when preoperative imaging suggests complete nodal response. Tracing methods include the placement of titanium clips [20] or radioactive seeds $[20,21]$ with or without preoperative wire placement or carbon particles [22]. At surgery post-NACT, these marked nodes are to be removed, and the various methods have each shown inherent advantages and disadvantages. Radioactive seeds, for example, may not be used in all countries due to radiation regulations, and may pose a problem when not retrieved at surgery; clips may migrate and not be detectable after months of NACT, and wires may exert significant discomfort in the patient. TAD may be performed by itself or in combination with an SLNB. It is agreed that SLNB plus TAD may yield significantly improved FNRs well within or below the range of known FNRs in the context of a negative SLNB in the adjuvant setting, namely $1.4-7 \%$ [20, 21]. Although favorable results have been published with the use of TAD, numerous questions still remain unresolved. These regard the reproducibility of the technique, the extent of surgery (number of resected nodes), and the clinical significance of a technically improved SLNB and decreased FNR.

Since the widely accepted FNR of $10 \%$ is an arbitrarily chosen cut-off, its clinical relevance should be briefly discussed. The FNR is defined as the number of false-negative results (SLNB-negative, ALND-positive) divided by all positive findings (SLNB- and/or ALND-positive). Of 474 patients in the SENTINA trial who converted from a clinically positive to a clinically negative axillary status after NACT and had a successful lymphatic mapping procedure, 248 (52.6\%) were ypN0 and 226 (47.7\%) ypN1. Assuming a 10,20 , or $30 \% \mathrm{FNR}$, the failure rate for the entire cohort would have been 5,10 , or $15 \%$, respectively. The clinical relevance of these figures is completely unclear due to the lack of outcome data; there is reason to hypothesize that FNR after NACT might not translate into a corresponding number of axillary failures when considering the observations reported after a negative SLNB in the adjuvant setting. The risk of the procedure to fail thus depends on 2 factors: FNR and the individual risk of axillary involvement after NACT, which clearly differs according to tumor subtype and the neoadjuvant treatment regimen applied. Patient selection should therefore be a valid tool to reduce the risk for individual failures of SLNB after NACT in $\mathrm{cN} 1$ patients. Tadros et al. [10] could show that $89.6 \%$ of 237 node-positive patients undergoing NACT and achieving a pCR in the breast were ypN0. Applying the calculations described above, this would translate into true failure rates as low as 1,2 , or $3 \%$.

Several nomograms have been developed to predict a complete nodal response for node-positive patients after NACT [23-25]. Kantor et al. [23] developed a model based on 19,115 node-positive patients undergoing NACT registered in the American National Cancer Data Base (NCDB). Young age, grade, intrinsic subtype, tumor histology, clinical N stage, and tumor response were predictive for $\mathrm{pN}$ conversion. Interestingly, complete tumor response in the breast showed the strongest association with nodal response (odds ratio: 20.37). Thus, individualized patient selection would 
probably contribute even more to the reduction of SLNB failures after NACT than technical improvement to reduce the FNR.

Missing outcome data are an important concern that prevents the routine use of SLNB after NACT in $\mathrm{CN} 1$ patients [26]. 2 recently published retrospective single-center studies provided survival data for $\mathrm{cN}+$ patients undergoing different types of axillary staging, axillary surgery, and RT. Galimberti et al. [4] reported on a limited retrospective cohort of 147 initially $\mathrm{cN}+$ patients who converted to $\mathrm{cN}-$ after NACT. SLNB was performed after NACT and was negative in 70 (47.6\%) patients; the remaining 77 patients with SLN metastases received ALND. Only in the latter group, 1 (1.3\%) axillary recurrence was observed. Survival according to SLN status differed only for those patients converting to ypT0 $(\mathrm{n}=38, \mathrm{p}=$ 0.03 ), with an OS of $86.3 \%$ for all patients initially $\mathrm{cN}+$. While HER2-positive and TN tumor subtypes had a significantly higher hazard ratio for distant recurrence after a median follow-up of 61 months, this was not true for SLN status or axillary surgery. Martelli et al. [27] reported on $243 \mathrm{cN}+$ patients, $46.9 \%$ of whom had converted to ypN0 after NACT. Patients received ALND ( $\mathrm{n}=85$, $35 \%)$, SLNB and ALND ( $\mathrm{n}=115,47.3 \%)$, or SLNB only $(\mathrm{n}=43$, $17.7 \%$ ). Those $200 \mathrm{cN}+$ patients who converted to ypN0 after NACT had a 10 -year OS of $86.9 \%$, to be compared with $72.5 \%$ for those with remaining axillary disease (ypN1).

\section{Future Perspectives}

The extent of breast surgery is already adapted to the degree of response to NACT; in the axilla, similar efforts to individualize treatment are currently ongoing. 2 US trials are underway investigating limited treatment strategies after NACT in a randomized fashion. The Alliance A011202 trial (ClinicalTrials.gov identifier: NCT01901094) focuses on clinically node-positive patients who convert to clinical node negativity after NACT. TAD is not part of the protocol, but SLNB is performed after NACT. Solely in the case of metastatic SLN deposits, patients are randomized to ALND plus regional nodal RT (no irradiation of the dissected axilla) or no ALND but axillary and regional nodal RT. For those patients who convert to being not only clinically but also pathologically node negative (ypN0), the NSABP B-51/RTOG 1304 trial (ClinicalTrials. gov identifier: NCT01872975) randomizes to either regional nodal RT or no RT apart from whole-breast irradiation after BCS. Axillary surgery confirming node negativity is not specified and TAD not implemented.

Genomic testing might be a future tool to select patients with expected good nodal response to chemotherapy who then might be spared extensive axillary surgery. This approach is currently tested prospectively in the Austrian AGO-35 trial which evaluates a genomic test developed at the MD Anderson Cancer Center. In the validation cohort, the test correctly predicted complete nodal response in $70 \%$ of the patients ( $95 \%$ confidence interval 50-86) [28].

More survival data are urgently needed, and, hopefully, followup data from the large SLNB trials in $\mathrm{cN}+$ patients receiving NACT will soon be available. There is clearly a need for European trials attempting to individualize nodal staging strategies and treatment both in terms of surgery and RT in patients presenting with nodepositive breast cancer and converting to node negativity after NACT. Modern trials should focus on survival outcomes and individual risk assessment and aim to prove that the extent of axillary treatment may be safely reduced based on reliable axillary staging methods such as SLNB with or without TAD post-NACT.

\section{European Trials in Progress}

Several European trials addressing the issue of axillary management in patients undergoing NACT are about to start recruiting in the very near future.

NEO NOD 2 is an Italian trial with $850 \mathrm{~T} 1-3 \mathrm{cN} 1$ patients who undergo NACT. The trial evaluates if ALND can be omitted in patients with micrometastases in the post-NACT SLN (130 ypN0(mi) patients needed). The study is planned to start in September 2018. Endpoints are OS, DFS, and distant DFS.

The French GANEA 3 trial is a prospective multicenter cohort study. $375 \mathrm{cN} 1$ patients treated with NACT will be included. Patients undergo TAD, SLNB, and ALND. Endpoint are the FNRs of the procedures applied.

The British ATNEC trial is a prospective multicenter randomized trial that will include 2,465 T1-3 N1 breast cancer patients treated with NACT. Patients undergo BCS or mastectomy and TAD with SLNB. At least 3 nodes have to be removed. Patients with 1-2 positive post-NACT SLNs will be randomized to undergo RT or ALND. Patients with ypN0 status will receive either RT or ALND in one arm and no further regional treatment in the second arm. Endpoint is DFS at 5 years.

The Swiss TAXIS trial is a prospective multicenter randomized trial with a planned accrual of 1,500 patients within 5.4 years. Breast cancer patients with stage II/III disease confirmed in the primary tumor and the lymph nodes $(\mathrm{cN} 1, \mathrm{ycN} 1)$ are eligible for the study. Patients are treated either with primary surgery or NACT. They are randomized to undergo ALND or the exclusive resection of clinically apparent disease (excision of suspicious clipped nodes) with consecutive RT to the axilla. Endpoint is DFS after 5 years.

\section{Disclosure Statement}

There is no conflict of interest to declare.
Kühn/Classe/Gentilini/Tinterri/Peintinger/de Boniface 


\section{References}

1 Classe JM, Bordes V, Campion L, et al: Sentinel lymph node biopsy after neoadjuvant chemotherapy for advanced breast cancer: results of Ganglion Sentinelle et Chimiothérapie Neoadjuvante, a French prospective multicentric study. J Clin Oncol 2008;27:726-732.

2 Hunt KK, Yi M, Mittendorf EA, et al: Sentinel lymph node surgery after neoadjuvant chemotherapy is accurate and reduces the need for axillary dissection in breast cancer patients. Ann Surg Oncol 2009;250:558566.

3 Classe J-M, Loaec C, Alran S, et al: Sentinel node detection after neoadjuvant chemotherapy in patien without previous axillary node involvement (GANEA 2 trial): follow-up of a prospective multi-institutional cohort. SABCS 2016;abstr S2-07.

4 Galimberti V, Ribeiro Fontana SK, Maisonneuve P, et al: Sentinel node biopsy after neoadjuvant treatment in breast cancer: five-year follow-up of patients with clinically node-negative or node-positive disease before treatment. Eur J Surg Oncol 2016;42:361-368.

5 Giordano SH, Elias AD, Gradishar WJ: NCCN guideline updates: breast cancer. J Natl Compr Canc Netw 2018;16:605-610.

6 Wöckel A, Albert US, Janni W, et al: The screening, diagnosis, treatment and follow-up of breast cancer. Dtsch Arztebl Int 2018;115:316-323.

7 Liedtke C, Jackisch C, Thill M, et al.; on behalf of the AGO Breast Committee: AGO recommendations for the diagnosis and treatment of patients with early breast cancer: update 2018. Breast Care 2018;13:196208.

8 www.swebcg.se.

9 Curigliano G, Burstein HJ, Winer E, et al: De-escalating and escalating treatments for early stage breast cancer: the St. Gallen International Expert Consensus Conference on the Primary Therapy of Early Breast Cancer 2017. Ann Oncol 2017;28:1700-1712.

10 Tadros AB, Yeng WT, Krishnamurthy S, et al: Identification of patients with documented pathologic complete response in the breast after neoadjuvant chemotherapy for omission of axillary surgery. JAMA Surg 2017;152:665-670.
11 Siso C, Esgueva R, Rodriguez M, et al: Her2 positive and triple negative breast cancer patients with clinically negative nodes at diagnosis and breast pathologic complete response may spare axillary surgery after neoadjuvant treatment. Eur J Cancer 2018;92(suppl 3):S21.

12 Van der Noordaa M, van Duijnhoven F, Cuijpers V, et al: Sentinel lymph node biopsy after neoadjuvant systemic therapy in selected breast cancer patients with clinical node-negative disease. Eur J Cancer 2018; 92(suppl 3):14.

13 Van Deurzen C, Vriens B, Tjan-Heijnen V, et al: Accuracy of sentinel node biopsy after neoadjuvant chemotherapy in breast cancer patients: a systematic review. Eur J Cancer 2009;45:3124-3130.

14 Van Nijnatten TJA, Schipper RJ, Lobbes MBI, et al: The diagnostic performance of sentinel lymph node biopsy in pathologically confirmed node positive breast cancer patients after neoadjuvant systemic therapy: a systematic review and meta-analysis. Eur J Surg Oncol 2015;41:1278-1287.

15 Kuehn T, Bauerfeind I, Fehm T, et al: Sentinel-lymphnode biopsy in patients with breast cancer before and after neoadjuvant chemotherapy (SENTINA): a prospective, multicentre cohort study. Lancet Oncol 2013; 14:609-618.

16 Boughey J, Suman V, Mittendorf E, et al: Sentinel lymph node surgery after neoadjuvant chemotherapy in patients with node-positive breast cancer. The ACOSOG Z1071 (Alliance) clinical trial. JAMA 2013; 310:1455-1461.

17 Nieweg O, Jansen L, Valdes Olmos RA, et al: Lymphatic mapping and sentinel lymph node biopsy in breast cancer. Eur J Nucl Med 1999;26(suppl):S11S16.

18 Holmstrand Zetterlund L, Frisell J, Zouzos A, et al: Swedish prospective multicenter trial evaluating sentinel lymph node biopsy after neoadjuvant systemic therapy in clinically node-positive breast cancer. Breast Cancer Res Treat 2017;163:103-110.

19 Boileau JF, Poinier B, Basik M et al: Sentinel node biopsy after neoadjuvant chemotherapy in biopsy proven node-positive breast cancer. J Clin Oncol 2014;33: 258-264.
20 Caudle AS, Yang WT, Krishnamurthy S, et al: Improved axillary evaluation following neoadjuvant therapy for patients with node-positive breast cancer using selective evaluation of clipped nodes: implementation of targeted axillary dissection. J Clin Oncol 2016;34: 1072-1078.

21 Donker M, Straver ME, Wesseling J, et al: Marking axillary lymph nodes with radioactive iodine seeds for axillary staging after neoadjuvant systemic treatment in breast cancer patients. the MARI procedure. Ann Surg 2015;261:378-382.

22 Choy N, Lipson J, Porter C, et al: Initial results with preoperative tattooing of biopsied axillary lymph nodes and correlation to sentinel lymph nodes in breast cancer patients. Ann Surg Oncol 2015;22:377382 .

23 Kantor O, Sipsy LM, Yao K, James TA: A predictive model for axillary node pathologic complete response after neoadjuvant chemotherapy for breast cancer. Ann Surg Oncol 2018;25:1304-1311.

24 Kim JY, Hyung SP, Kim S, et al: Prognostic nomogram for prediction of axillary pathologic complete response after neoadjuvant chemotherapy in cytologically proven node-positive breast cancer. Medicine (Baltimore) 2015;94:e1720

25 Schipper RJ, Moosdorff M, Nelemans PJ, et al: A model to predict pathologic complete response of axillary lymph nodes to neoadjuvant chemo (immuno) therapy in patients with clinically node positive breast cancer. Clin Breast Cancer 2014;14:315-322.

26 Lyman GH, Temis S, Edge SB, et al: Sentinel lymph node biopsy for patients with early stage breast cancer: American Society of Clinical Oncology Clinical Practice Guideline update. J Clin Oncol 2014;32:32:1365-1383.

27 Martelli G, Miceli R, Folli S, et al: Sentinel node biopsy after primary chemotherapy in cT2 N0/1 breast cancer patients: long-term results of a retrospective study. Eur J Surg Oncol 2017;43:2012-2020.

28 Peintinger F, Kühn T, Schermann C, et al: AGO Austria-35: Prospektive Validierung der genomischen Signaturen zur Erfassung der Chemosensitivität der axillären Lymphknoten nach neoadjuvanter Chemotherapie beim HER2 negativem Mammakarzinom. Senologie 2015;12:A113. 